\title{
BIODETERIORAÇÃO DA MADEIRA TRATADA DE Eucalyptus sp. COMERCIALIZADA NO SUDESTE PARAENSE
}

\author{
BIODETERIORATION OF TREATED WOOD OF Eucalyptus $\mathbf{s p .}$ \\ COMMERCIALIZED IN PARAENSE SOUTHEAST
}

\author{
Laise De Jesus Dos Santos ${ }^{1}$; Thiago dos Reis Lima²; Felipe Valente da Cruz ${ }^{3}$; Luiz Eduardo \\ de Lima Melo ${ }^{4}$ J Javan Pereira Motta ${ }^{5}$
}

DOI: https://doi.org/10.31692/978-65-991061-7-0.83-92

\section{RESUMO}

O desenvolvimento da humanidade está fortemente atrelado ao uso das florestas, esta necessidade foi suprida por muitos anos quase que exclusivamente por madeira de floresta nativa, que foi explorada de modo predatório durante o século XIX. Novas legislações passaram a defender as florestas nativas, obrigando assim o mercado a encontrar novas alternativas para suprir a demanda, encontrando a solução em florestas plantada, como as do gênero Eucalyptus, uma árvore que apresenta rápido crescimento, alta capacidade de adaptação e grande variável de aplicação em diferentes setores. O trabalho avaliou a biodeterioração por térmitas, da madeira tratada de Eucalyptus spp., frequentemente comercializada no sudeste do Pará na forma de moirões. A madeira utilizada foi obtida de 1 moirão tratado, adquirido no município de Marabá, comercializado como madeira tratada de Eucalyptus spp., imunizada quimicamente com o CCA, de acordo com informações do comércio local. A pesquisa foi conduzida no Laboratório de Ciência e Tecnologia da Madeira, da Universidade do Estado do Pará, no município de Marabá, PA. Foram definidas 3 regiões amostrais no sentido longitudinal do moirão tratado, sendo elas base, meio e topo. De cada região foram retirados 6 discos com $15 \mathrm{~cm}$ de altura da seção transversal, totalizando 18 discos, além disso, se definiu dois tipos de madeira: 1 - cerne (cerne interno); 2 - mista (alburno e cerne periférico). Retirou-se de cada disco 16 corpos de provas, que totalizou 288 corpos de prova, com dimensões de 0,60 x 2,54 x 10,0 cm (tangencial x radial x longitudinal) seguindo a ASTM D 2017 (2005), a análise da resistência à biodeterioração da madeira tratada seguiu recomendação da norma ASTM D3345-74 (1999). Observou-se que aposição longitudinal não interferiu na resistência aos cupins de solo da madeira tratada. Verificou-se que o tipo de madeira tratada e mista, influenciou no comportamento da perda de massa e desgaste. Portanto, se constatou que a madeira de Eucalyptus sp. tratada comercializada no município de Marabá possui a proteção necessária para impedir danos causados por cupins de solo Nasutitermes corniger.

Palavras-Chave: Preservação da madeira, Marabá, moirão tratado, Nasutitermes spp.

\section{ABSTRACT}

The development of mankind is strongly tied to the use of forests, this need was supplied for many years almost exclusively by native forest wood, which was exploited predatoryly during the nineteenth century. New legislation began to defend native forests, thus obliging the market to find new alternatives to meet the demand, finding the solution in planted forests, such as those of the genus Eucalyptus, a tree that presents rapid growth, high adaptability and great variable of different sectors. The work evaluated the biodeterioration by termites of the treated wood of Eucalyptus spp, often marketed in Southeastern of State Pará, Brazil in the form of fence posts. The wood used was obtained from 1 treated fence posts, acquired in the municipality of Marabá, marketed as treated wood of Eucalyptus sp. chemically immunized with the CCA, according to local trade information. The research was conducted at the Laboratory of Science and Technology of Madeira, State University of Pará, in the 
municipality of Marabá, PA. Three sample regions were defined in the longitudinal direction of the treated fence posts, being base, middle and top. From each region 6 discs with $15 \mathrm{~cm}$ of cross section were removed, totaling 18 discs, in addition, two types of wood were defined: 1 - heartwood (inner heartwood); 2 - mixed (sapwood and peripheral heartwood). From each disk, 16 test pieces were collected, totaling 288 specimens measuring $0.60 \times 2.54 \times 10.0 \mathrm{~cm}$ (tangential x radial x longitudinal) following ASTM D 2017 (2005), the analysis of the resistance to biodeterioration of treated wood followed the recommendation of ASTM D334574 (1999). It was observed that longitudinal apposition did not interfere in the soil termite resistance of the treated wood. It was verified that the type of treated and mixed wood, influenced in the behavior of the loss of mass and wear. Therefore, it was found that the wood of Eucalyptus sp. treated commercialized in the municipality of Marabá has the necessary protection to prevent damage caused by termites of Nasutitermes corniger.

Keywords: Treated wood, Marabá State of Pará, Brazil, fence posts, Nasutitermes corniger.

\section{INTRODUÇÃO}

Os Eucalyptus spp. passaram a ser cultivados comercialmente no Brasil em 1904, com a finalidade energética de fornecer lenha às locomotivas da Companhia Paulista de Estradas de Ferro (PAES, 2002). Atualmente, o Brasil possui uma das maiores áreas com plantações desse gênero no mundo, estimado em 5,7 milhões de hectares. Sendo que sua madeira é destinada à produção de celulose e papel, moirões, painéis de madeira, pisos laminados, carvão vegetal e biomassa (IBÁ, 2017).

Motivado por sua composição química e estrutura anatômica, a madeira torna-se suscetível a deterioração por diversos organismos biodeterioradores (SILVA et al., 2014). Segundo Paes (2002) árvores que apresentam um rápido crescimento, como é o caso do Eucalyptus spp. têm, em comum, menos resistência natural.

A madeira tratada de Eucalyptus, em especial aquela que tem a finalidade de atender a demanda de moirões é derivada de lenho jovem (PAES et al., 2012). Bem como madeira denominada alburno ou brancal de peças de maiores diâmetros, acima de $20 \mathrm{~cm}$ e diâmetro. Verifica-se que essa madeira é mais susceptível ao ataque e aos danos significativos à estrutura do lenho pelos insetos xilófagos, como cupins e brocas. Os cupins são os insetos mais severos que atacam e destroem a madeira pelo mundo (TREVISAN et al., 2007). Para esse tipo de madeira, em que não há defesa natural contra a ação desses xilófagos, se faz necessário imunizar industrialmente a madeira, no qual se utiliza preservativos químicos.

Um dos produtos mais empregados no tratamento industrial preservativo da madeira é o Arseniato de Cobre Cromatado, conhecido como CCA (ARAUJO, 2012). Segundo Arantes et al. (2017) esse produto é uma combinação que envolve cobre $(\mathrm{Cu})$, cromo $(\mathrm{Cr})$ e arsênio (As). Ponderando que não há um preservativo para madeira que não provoque danos à saúde humana e que seja altamente eficaz contra a deterioração biológica, o CCA é o 
preservativo que proporciona os melhores resultados quanto a ação, segurança e a relação benefício e custo (ARAUJO et al., 2012). O controle de térmicas é importante por estar ligado diretamente ao prejuízo econômico que estes organismos podem provocar quando atacam a madeira (LOPES et al., 2017).

Diante do exposto, o presente trabalho objetivou avaliar a qualidade da madeira tratada de Eucalyptus spp., comercializada no sudeste paraense, com foco no município de Marabá, por meio da avaliação do nível de danos causados por térmitas, denominados cupins de solo, do tipo arborícola. Visto que, desde 2012 essa madeira tratada foi introduzida na região, com a possibilidade de substituir a madeira nativa e reduzir da exploração dos maciços florestais do bioma Amazônico.

\section{FUNDAMENTAÇÃO TEÓRICA}

Não existem estatísticas que apontem o número de moirões de cerca utilizados no Brasil. Contudo, considerando que $65 \%$ do volume total de madeira tratada no País tem como destino o uso como mourões de eucaliptos, é plausível estimar que sua produção média anual seja na ordem de 40 milhões de unidades (GERALDO, 2008).

Os cupins agridem a madeira por meio de um protozoário que vive no seu intestino em completa interação (FLORIAN, 2013). A biodeterioração da madeira é o dano causado por agentes naturais como os microrganismos, insetos e os xilófagos marinhos. As avarias realizadas pelos térmitas podem ser irremediáveis, chegando a provocar colapsos na madeira (BAHIA, 2015; MARTINS, 2009).

Os cupins Nasutitermes corniger possuem coloração escura, constroem túneis e galerias que vão desde o ninho até o chão, não apresentam preferência por espécie de madeira, atacando madeiras com maior e menor densidade, porém preferem alburno em detrimento ao cerne. São conhecidos por causarem grandes danos à madeira atacada, um fator que influência nesta escolha é o grau de decomposição que a madeira apresenta (SILVA, 2008).

\section{METODOLOGIA}

O trabalho foi conduzido no Laboratório de Ciência e Tecnologia da Madeira do curso de Engenharia Florestal, da Universidade do Estado do Pará, Campus VIII Marabá. A madeira foi proveniente do comércio local, do município de Marabá, sudeste do Estado do Pará, localizado na latitude $05^{\circ} 22^{\prime} 07^{\prime}$ ' S e longitude $49^{\circ} 07^{\prime} 04^{\prime}$ ' W. Selecionou-se um moirão tratado industrialmente com Arseniato de Cobre Cromatado - CCA, de acordo com informações do comércio. O moirão pertencia a classe diamétrica de 20 a $22 \mathrm{~cm}$ e 2,83 m de 
comprimento.

\section{Confecção dos corpos de prova}

Foram retirados com o auxílio de um motosserra 18 discos da seção transversal com $15 \mathrm{~cm}$ de altura no sentido longitudinal (base, meio, topo). Vale ressaltar que de cada extremidade, base e topo, foram descartados $6,5 \mathrm{~cm}$ de madeira, para evitar erros de leitura de umidade. Foram definidas 3 regiões amostrais, sendo a base (discos de 1 a 6), meio (discos de 7 a 12) e topo (discos de 13 a 18). De cada disco foram retirados 16 corpos de prova, totalizando 288, sendo 144 corpos de prova de cerne (cerne interno) e 144 de madeira mista (alburno e cerne periférico). Os corpos de prova foram confeccionados seguindo recomendações da ASTM D 2017 (2005), com dimensões de 0,60×2,54 × 10,0 cm (tangencial x radial x longitudinal). Os corpos de provas foram todos lixados, para eliminar defeitos, secados a temperatura de $103 \pm 2^{\circ} \mathrm{C}$, até atingirem a massa constante, e a pesagem foi realizada em uma balança de precisão $(0,01 \mathrm{~g})$.

\section{Ensaio de preferência alimentar}

Os corpos de provas foram colocados em uma caixa d'água de $250 \mathrm{~L}$, enterrados em até $1 / 3$ da dimensão longitudinal em areia úmida. Os corpos de prova foram arranjados de acordo com um delineamento em blocos casualizados, totalizando oito fileiras de corpos de prova dentro da caixa, no espaçamento de $6,3 \mathrm{~cm}$ entre as fileiras e 4,2 $\mathrm{cm}$ entre as amostras.

A colônia de térmitas utilizada no experimento foi coletada em uma área nas proximidades do Campus VIII da Universidade do Estado do Pará, localizado no município de Marabá, PA. Os corpos de prova permaneceram expostos à ação de cupins durante 45 dias, em local com temperatura e umidade relativa do ar ambiente. A análise da biodeterioração seguiu as recomendações da ASTM D3345-74 (1999).

A análise estatística foi em delineamento em blocos casualizados - DBC, por meio de um fatorial 2 × $3 \times 6$, sendo tipo de madeira (2), posição longitudinal (3) e número de disco por posição (6), a 5\% de significância, em que se analisou a influência de cada fator na resistência da madeira do moirão tratado as térmitas ao longo do sentido longitudinal. Sendo que essa característica foi avaliada por meio da perda de massa (\%) e desgaste (nota) conforme a Tabela 1, de acordo com Motta et al. (2013) e Paes et al. (2015).

Os valores em porcentagem da perda da massa foram transformados em arcsen $\sqrt{\text { Perda da massa/100 }}$ e o desgaste (nota) em $\sqrt{\text { Nota }+0,5}$ para permitir a homogeneidade das variâncias, de acordo com Stell e Torrie (1980). 
Tabela 1. Avaliação do desgaste da madeira.

\begin{tabular}{cc}
\hline Desgaste & Notas \\
\hline Sadio, permitindo escarificações superficiais & 10 \\
Ataque superficial & 9 \\
Ataque moderado, havendo penetrações & 7 \\
Ataque intensivo & 4 \\
Falha, havendo ruptura dos corpos de prova & 0 \\
\hline
\end{tabular}
Fonte: ASTM D - 3345 (ASTM, 2005).

\section{RESULTADOS E DISCUSSÕES}

O comportamento e o efeito dos fatores principais, como posição longitudinal, número de disco e tipo de madeira na perda de massa (\%) da madeira do moirão tratado de Eucalyptus sp. encontra-se na Figura 1.

Figura 1 - Efeito dos fatores principais na perda de massa (\%) da madeira do moirão tratado de Eucalyptus sp.

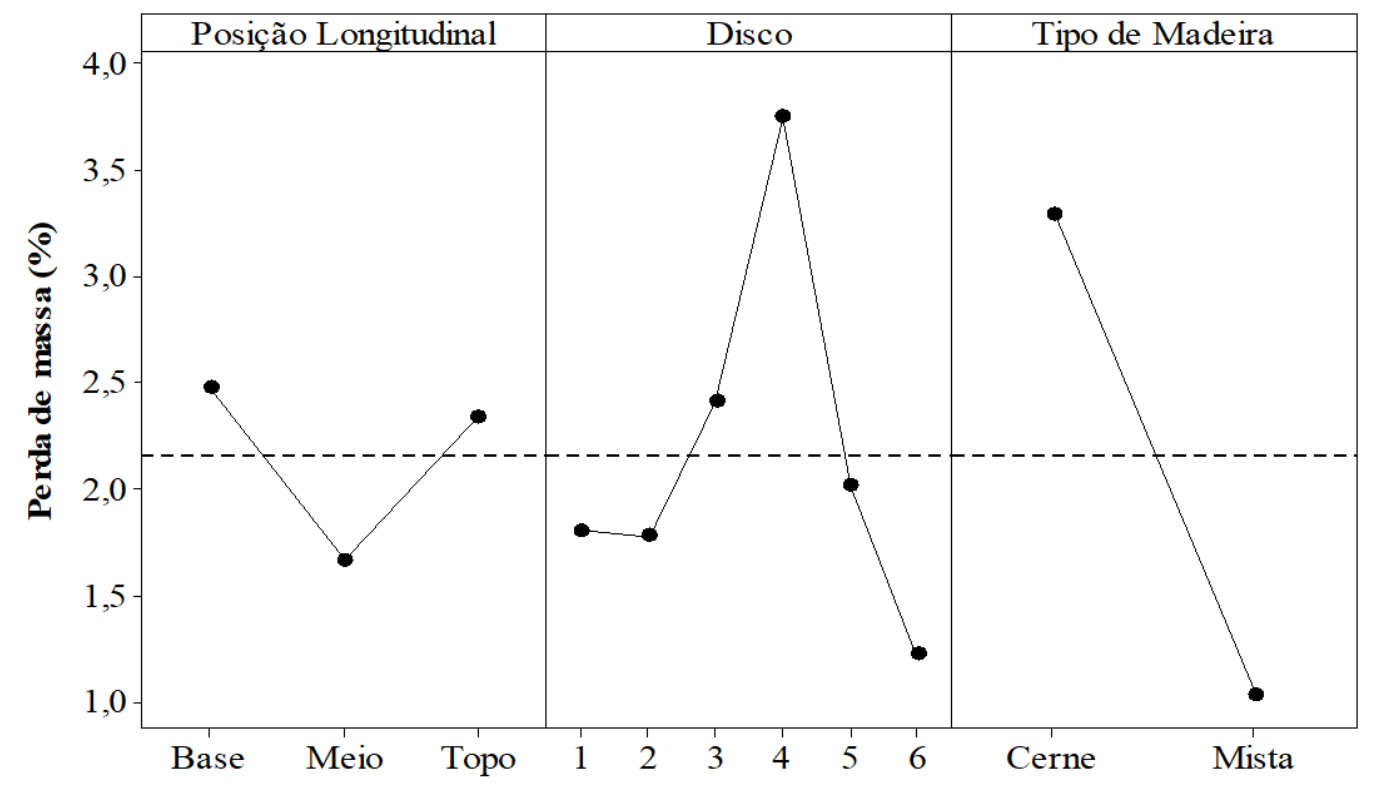

Verifica-se na Figura 1 que a perda de massa (\%) causada pelas térmitas no sentido longitudinal da madeira obteve maior valor na base $2,5 \%$, seguido no topo com aproximadamente $2,3 \%$ e o meio com cerca de $1,7 \%$. O resultado de menor de perda de massa identificado na posição longitudinal denominada meio indicou que o tratamento industrial utilizado no moirão foi mais eficiente nessa região, contrariando resultados obtidos por outros autores (CALIL JUNIOR; BRITO 2010).

Verifica-se na Figura 1 que a perda de massa (\%) nos discos 1 e 2 foi de aproximadamente 1,8\%; no disco 3 valor de $2,5 \%$, e no 4 foi observado o maior valor de perda de massa, cerca de 3,8\%, o disco 5 obteve perda de massa igual a $2,0 \%$ e o disco 6 obteve o menor valor de perda de massa com 1,3\%. Na Figura 1, constata-se que o comportamento da perda de massa entre os tipos de madeira foi significativo. Na avaliação de 
perda de massa os corpos de prova confeccionados com madeira do cerne interno obtiveram maior perda de massa, equivalente a 3,3\%, enquanto os corpos de prova misto, confeccionados com madeira de alburno e cerne periférico não sofreram ataque relevante, resultando em um valor de $1,0 \%$, podendo ser justificado essas menores percas de massa por eles terem uma boa porcentagem de madeira tratada (Figura 1). Lopes et al. (2017) destacaram em seu trabalho menor perda de massa nos corpos de prova retirados do alburno, devido a este conter maior penetração do produto preservativo CCA, enquanto o cerne apresentou uma elevada perda de massa devido a menor penetração do produto preservativo.

Figura 2 - Interação dos fatores principais na perda de massa (\%) da madeira do moirão tratado de Eucalyptus sp.

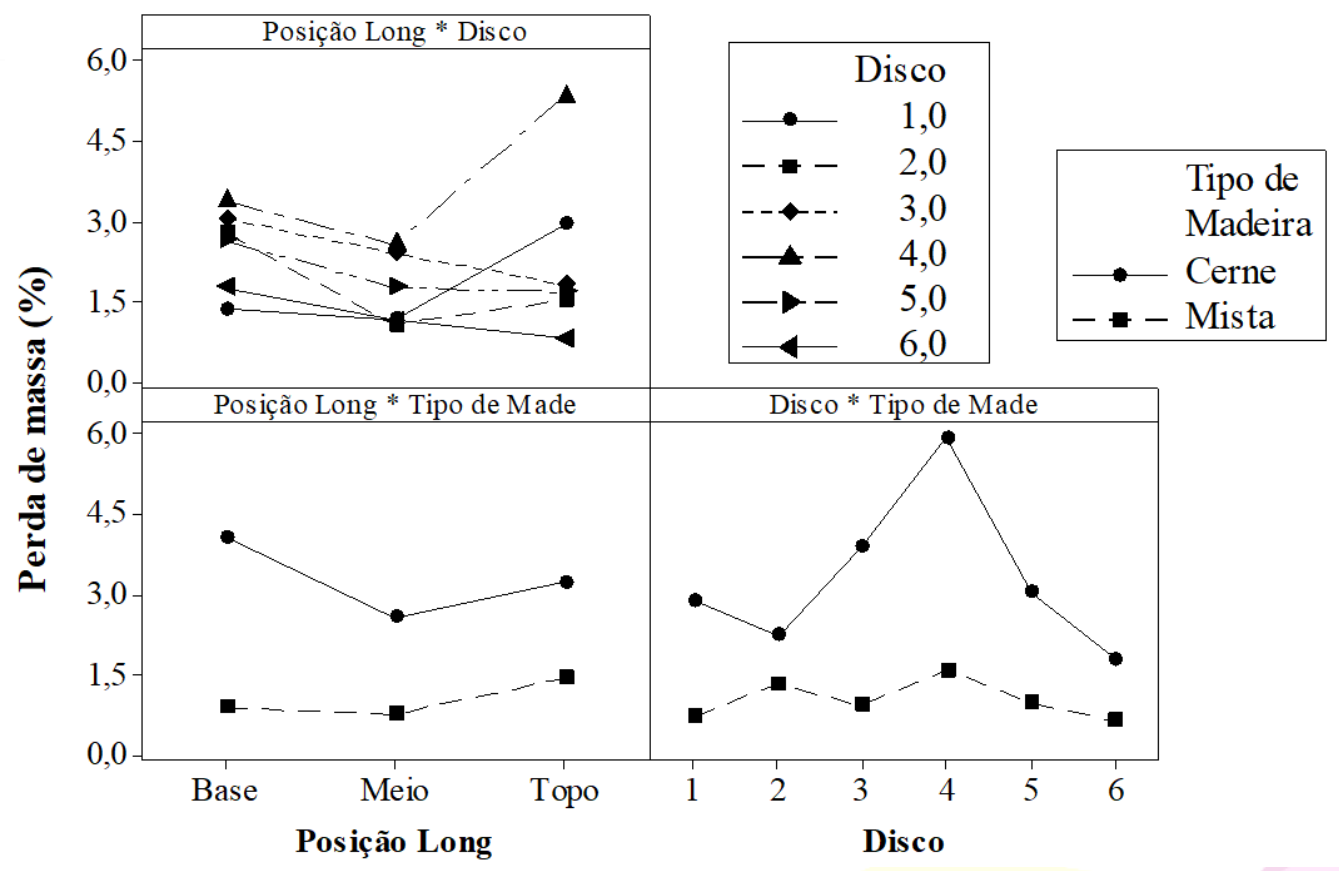

A interação da posição longitudinal e disco (Figura 2) mostrou que os discos do meio demonstraram menor taxa de perda de massa em relação a base e topo. Verificou-se que os discos 1 e 4, obtiveram a maior perda de massa 3,0\% e 5,8\%, respectivamente. Observou-se também que os discos que mais resistiram ataque das térmitas foram o 6 e o 2, com cerca de $1 \%$ e $1,7 \%$ de perda de massa, respectivamente.

A interação da posição longitudinal e tipo de madeira verificou-se que o cerne presente na base apresentou maior perda de massa, sendo o valor médio igual a 4,4\%, seguido de topo e meio, com respectivos valores de 3,1\% e 2,9\%. Relacionado ao tipo de madeira mista, comprovou-se que o topo obteve perda de massa igual a 1,5\%, e meio e base com valores próximos de $1,3 \%$ (Figura 2).

Na Figura 2, a interação do disco com tipo de madeira os maiores valores de perda de 
massa no cerne ocorreram no disco $4(6,0 \%)$, disco $3(4,3 \%)$ e disco $5(3,0 \%)$, seguido dos discos 1,2 e 6 , com respectivos valores de 2,8\%, 2,0\% e 1,5\%. Foi observado que o disco 6 de madeira mista foi o que obteve o menor valor de perda de massa, com menos de $1,0 \%$, contudo o disco 4 teve $1,5 \%$ de perda de massa.

O comportamento e o efeito dos fatores principais, como posição longitudinal, número de disco e tipo de madeira no desgaste (nota) da madeira do moirão tratado de Eucalyptus sp. encontra-se na Figura 3.

Ao analisar desgaste dos corpos de prova foi possível verificar que na posição longitudinal, na base foi onde ocorreu o maior índice de desgaste comprovado com a média de nota 8,7, seguido pelo meio com nota de 9,3 e o topo com menor desgaste, com a nota 9,5 (Figura 3).

Figura 3 - Efeito dos fatores principais no desgaste (nota) da madeira do moirão tratado de Eucalyptus sp.

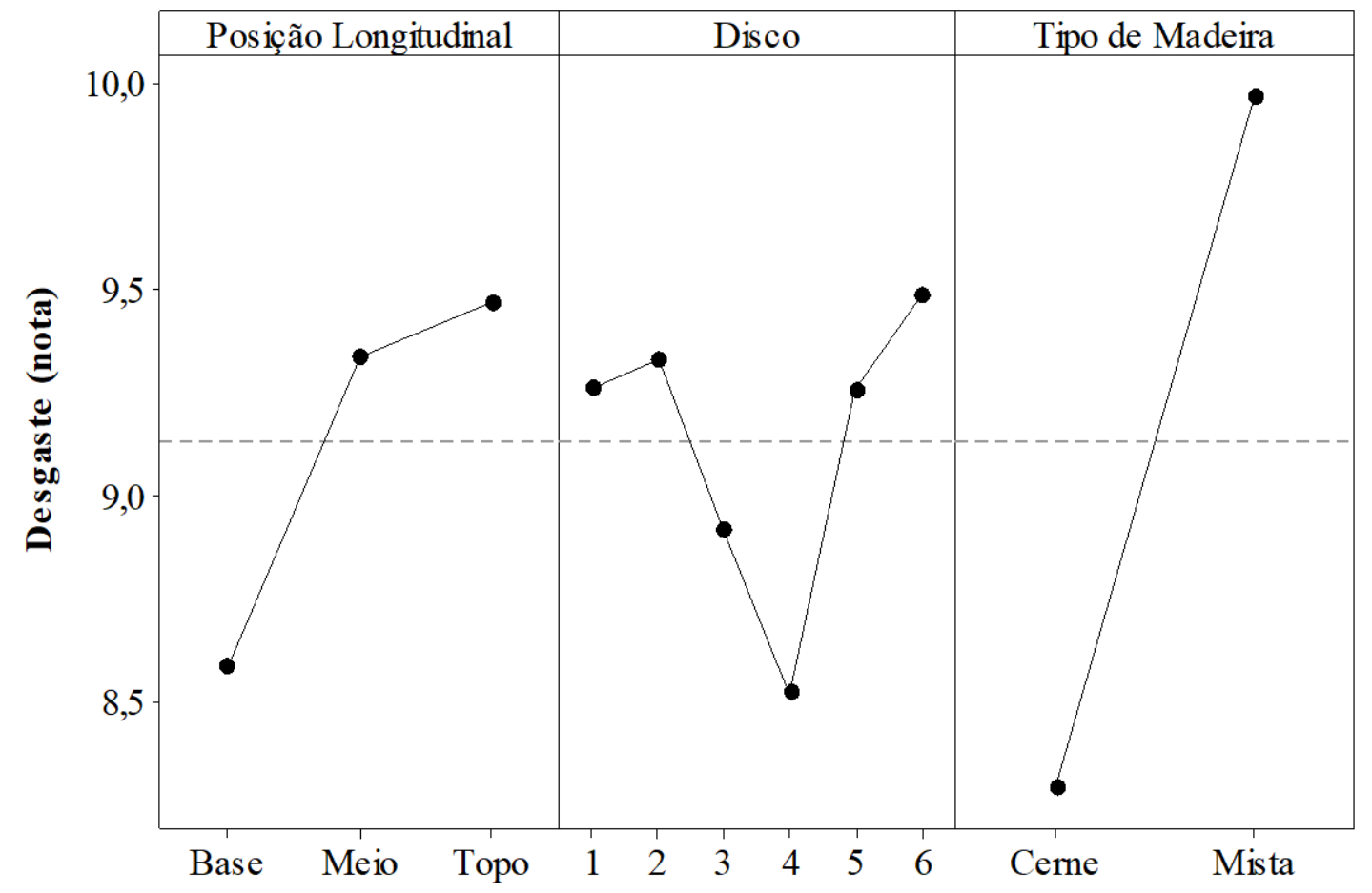

O desgaste nos 6 discos (Figura 3), indicou que o disco 4 obteve a menor nota com 8,3 , mostrando que ele foi o mais atacado, seguido pelo disco 3 com nota 8,9 , discos 5 e 1 com valores aproximados de 9,3 e juntamente com a nota 9,4 do disco 2 , visto que a maior nota obtida foi do disco 6 com 9,5.

A Figura 3 indica que o cerne foi o que apresentou um maior nível de desgaste obtendo nota abaixo de 8,5, já a madeira mista apresentou menor nível de desgaste atingindo nota de aproximadamente 9,9. Araújo, Magalhães e Oliveira (2012) constataram trabalhando com Corymbia citriodora tratada com CCA que as estacas tratadas ficaram livres de ataque de 
xilófagos, enquanto as estacas não tratadas foram fortemente atacadas.

O comportamento da interação dos fatores principais no desgaste (nota) da madeira do moirão tratado de Eucalyptus sp. encontram-se na Figura 4. A interação de desgaste relacionada a posição longitudinal e os discos, mostrou que em média o menor desgate foi obtido na posição do meio. Conforme a Figura 4, em relação a posição longitudinal e os tipos de madeira, as melhores notas alcançadas foram da madeira mista, mostrando que base, meio e topo tiveram notas médias iguais a 7,1; 8,8; e 9,0; respectivamente.

Figura 4 - Interação dos fatores principais no desgaste (nota) da madeira do moirão tratado de Eucalyptus sp.

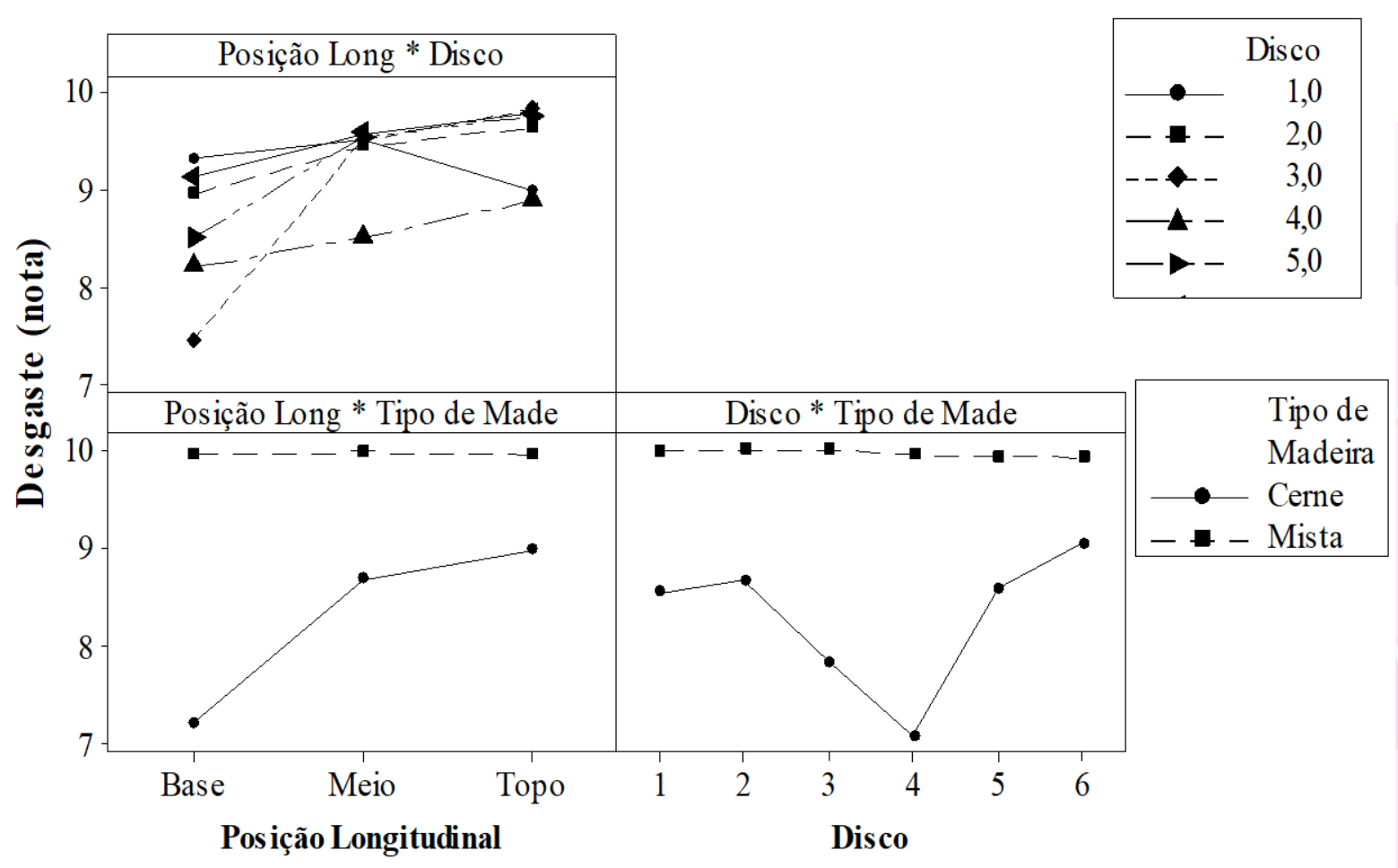

A interação entre discos e o tipo de madeira, comprovou que a madeira mista obteve as maiores notas (Figura 4). O cerne obteve as menores notas, que indicou sua menor resistência e maior desgaste ao ataque dos cupins, em que o disco 4 foi o mais atacado pelas térmitas, seguido pelos discos $3,1,2,5$ e 6 , recebendo as notas 7,0;8,0;8,7;8,9;8,9 e 9,0, respetivamente.

\section{CONCLUSÕES}

No sentido longitudinal, a posição meio foi a que apresentou maior resistência ao ataque dos cupins de solo $N$. corniger. O tipo de madeira determinou o comportamento da resistência aos cupins de solo $N$. corniger, sendo que o cerne, madeira não tratada, foi em geral susceptível ao ataque dos xilófagos. 
Pode se afirmar que o moirão tratado de Eucalyptus sp. comercializado em Marabá, no sudeste do Estado do Pará, possui a resistência necessária ao ataque dos cupins de solo Nasutitermes corniger.

\section{REFERÊNCIAS}

ARANTES, L. S. HEIN, R. G. SILVA, J. R. M. SOARES, V.C. Influência do tratamento preservativo com CCA-C na estabilidade dimensional da madeira de Eucalyptus. Scientia Forestalis, v.45, n.114, p.285-293, 2017.

ARAUjO, H. J. B. MAGAlHÃES, W. L. E. OLIVEIRA, L.C. Durabilidade de madeira de eucalipto citriodora (Corymbia citriodora (Hook.) K.D. Hill \& L.A.S. Johnson) tratada com CCA em ambiente amazônico. Acta Amazônica, v. 42, n.1, p. 49-58, 2012.

CARVALHO, D. E. et al. Principais agentes deterioradores de madeiras. Revista da Madeira, ed. n.134, 2013.

CALIL JUNIOR, C.; BRITO, L. D. Manual de projeto e construção de estruturas com peças roliças de madeira de reflorestamento. EESC-USP. p. 332, 2010.

GERALDO, F. C. Mourões para cercas: a renovação necessária. Revista da madeira, Curitiba, PR, n. 115, 2008.

INDÚSTRIA BRASILEIRA DE ÁRVORE IBÁ. 2017. Disponível em<https://iba.org/images/shared/Biblioteca/IBA_RelatorioAnual2017.pdf>. Acesso em: 08 de outubro de 2018.

LOPES, D. J. V. et al. Resistência biológica da madeira de eucalipto tratada com CCA-C a cupins subterrâneos em laboratório. Scientia Forestalis, v. 45, n. 115, p. 467-479, 2017.

MARTINS, S.F.M.F. 262f, 2009. Estruturas de Madeira - Inspeção e diagnóstico. Aplicação em Caso de Estudo. Dissertação (Mestrado em Engenharia Civil). Engenharia Civil - Área de Especialização Materiais, Reabilitação e Sustentabilidade da Construção Escola de Engenharia, Universidade do Minho, Portugal.

MOTTA, J. P. et al.. Resistência natural da madeira de Tectona grandis em ensaio de laboratório. Ciência Rural v. 43, p. 1393-1398, 2013.

PAES, J. B et al.. Qualidade de moirões de eucalipto tratados comercializados em três municípios do Espírito Santo. Floresta e Ambiente, Seropédica, v. 19, n.4, p. 475-482, 2012.

PAES, J. B. Resistência natural da madeira de Corymbia maculata (Hook.) K. D. Hill e L.A.S. Johnson a fungos e cupins xilófagos, em condições de laboratório. Revista Árvore, Viçosa, v. 26, n. 6, p. 761-767, 2002.

PAES, J. B. et al. Efeitos dos extrativos e da densidade na resistência natural de madeiras ao térmita Nasutitermes corniger. Cerne, v. 21, p. 569-578, 2015. 
SILVA, V. S.G. Comportamento de forrageamento de Nasutitermes corniger (MOTSCHULSKY) (ISOPTERA: TERMIDAE) e sua ocorrência em áreas urbanas. Ed. Campos dos Goytacazes - RJ, 2008.

SILVA, L. F. et al. Deterioração da madeira de Eucalyptus spp. por fungos xilófagos. Cerne, Lavras, v. 20, n. 3, p. 393-400, 2014.

TREVISAN, H. MARQUES, F. M. T. CARVALHO, A. G. Degradação natural de toras de cinco espécies florestais em dois ambientes. Floresta, v.38, n.1, 2008.

CARVALHO, D. E. et al. Variação dimensional da madeira tratada de Eucalyptus grandis e Eucalyptus cloeziana. Scientia Agraria Paranaensis, v.14, n.3, p.178-182, 2015. 